\title{
Analysis of Metal Flow Behavior and Residual Stress Formation of Complex Functional Profiles under High-Speed Cold Roll-Beating
}

\author{
Fengkui Cui, ${ }^{1,2}$ Yongxiang Su $\mathbb{D}^{1,2}{ }^{1,2}$ Kege Xie,, ${ }^{1,2}$ Wang Xiaoqiang, ${ }^{1,2}$ Xiaolin Ruan,,2 \\ and Fei Liu iD ${ }^{1,2}$ \\ ${ }^{1}$ School of Mechatronics Engineering, Henan University of Science and Technology, Luoyang 471003, China \\ ${ }^{2}$ Collaborative Innovation Center of Machinery Equipment Advanced Manufacturing of Henan Province, \\ Luoyang 471003, China
}

Correspondence should be addressed to Yongxiang Su; syxsuper@163.com

Received 16 October 2017; Accepted 29 March 2018; Published 3 May 2018

Academic Editor: Antonino Squillace

Copyright () 2018 Fengkui Cui et al. This is an open access article distributed under the Creative Commons Attribution License, which permits unrestricted use, distribution, and reproduction in any medium, provided the original work is properly cited.

\begin{abstract}
To obtain a good surface layer performance of the complex functional profile during the high-speed cold roll-beating forming process, this paper analyzed the metal plastic flow and residual stress-formed mechanism by using a theoretical model of the metal flow and residual stress generation. By using simulation software, the cold roll-beating forming process of a spline shaft was simulated and analyzed. The metal flow and residual stress formation law in the motion were researched. In a practical experiment, the changes in the grains in the spline tooth profile section and the residual stress distribution on the tooth profile were studied. A microcorrespondence relationship was established between the metal plastic flow and the residual stress generation. The conclusions indicate that the rate at which the metal flow decreases changes gradually at different metal layers. The residual stress value is directly related to the plastic flow difference. As the roll-beating speed increases, the uneven degree of plastic deformation at the workpiece surface increases, and the residual stress in the tooth profile is generally greater. At the same roll-beating speed, the rate change trend of the metal flow decreases gradually from the surface to the inner layer and from the dedendum to the addendum. The residual stress distribution on the surface of the tooth profile decreases from the dedendum to the addendum. These findings provide a basis and guidance for the controlled use of residual stress, obtaining better surface layer quality in the high-speed cold roll-beating process of the complex functional profile.
\end{abstract}

\section{Introduction}

The high-speed cold roll-beating precision formation of a complex functional profile is a sophisticated, efficient, green, and advanced manufacturing technology $[1,2]$. It uses the plasticity of metal materials to plastically deform the workpiece and maintain its shape after deformation. The metal plastic forming process enables the formation of a continuous fibrous tissue inside the workpiece to strengthen the material and improve the mechanical properties of the workpiece. However, metal plastic forming technology will inevitably produce residual stress in the workpiece interior $[3,4]$. On one hand, the residual stresses can reduce the expansion of surface microcracks and improve the workpiece fatigue strength; on the other hand, the stresses decrease the stability of the workpiece size [5-7]. In addition, recent studies on cold roll-beating formation have shown that changes in the surface roughness and surface hardening of the workpiece are closely related to the generation of residual stress [8-10]. Therefore, research on the formation, control, and utilization of residual stress in the high-speed cold rollbeating forming process has high academic significance and high value for engineering applications.

In previous research, Smirnov proposed the feasibility of using splines formed by cold roll-beating based on the experimental data. The inhomogeneity and complexity of 
the deformation extent and stress distribution in the forming process were verified in an experimental data analysis [11]. Kurz established a simulation model of plastic deformation in the cold roll-beating process that used finite element theory to calculate the value of forming force as well as stress and strain on the workpiece in the cold roll-beating process. A comparison of the simulation results and the experimental data showed that the simulation results were reliable [12]. Cui studied the dynamic response of the cold roll-beating process and the stress wave. The metal flow law and deformation mechanism of the involute spline were revealed from the macroperspective by analyzing the involute spline cold roll-beating process [13]. Zhang et al. used the slab method to analyze the deformation force and established an analytical equation by a simulation analysis [14].

In research on forming accuracy, Grob and Krapfenbauer studied the installation angle problem of a roll-beating wheel. The roll-beating wheel was fixed on the rolling head at the appropriate installation angle by adding a thrusting ring to improve the accuracy of the workpiece [15]. Cui modified the profile of the roller based on the relationship of the relative motion between the roll-beating wheel and the workpiece. A higher surface accuracy of the involute spline can be obtained by cold roll-beating than that can be obtained by milling [13].

In research on the forming surface quality, Weck et al. noted that the fatigue strength increased by $25 \%-35 \%$ due to the hardening layer on the surface of the cold roll-beating forming products by comparing the products of cold rollbeating and cutting [16]. Cui et al. studied the fiber tissue and the tooth surface hardness distribution of the spline in the process of cold roll-beating through a simulation analysis and experimental observation. The plastic deformation and the phenomenon of surface hardening during the process of cold roll-beating were explained from the microscopic perspective. The effect and properties of metal plastic deformation on the structure were analyzed. The effect of thermomechanical coupling on the metal surface hardening and the effect of forming parameters on the surface roughness of the workpiece in the cold roll-beating process were obtained $[8,9,17,18]$.

In summary, recent studies on the surface quality have concentrated mainly on examining the workpiece roughness, work hardening, and other factors. Little research has been done on the problem of residual stress being caused by uneven plastic deformation in the process of cold rollbeating to date. Therefore, the research on residual stress has important significance for determining the process parameters and further improving the surface quality of cold roll-beating.

In this paper, the metal plastic flow law of spline shafts in the process of cold roll-beating was studied based on the law of minimum resistance and volume invariance. The effect of uneven extent of the plastic deformation with different cold roll-beating speeds on the overall workpiece and the same rotation speed at different parts of the spline tooth profile was analyzed. The forming process of cold roll-beating was simulated, and the displacement of nodes was tracked by finite element software to verify the theoretical part of this

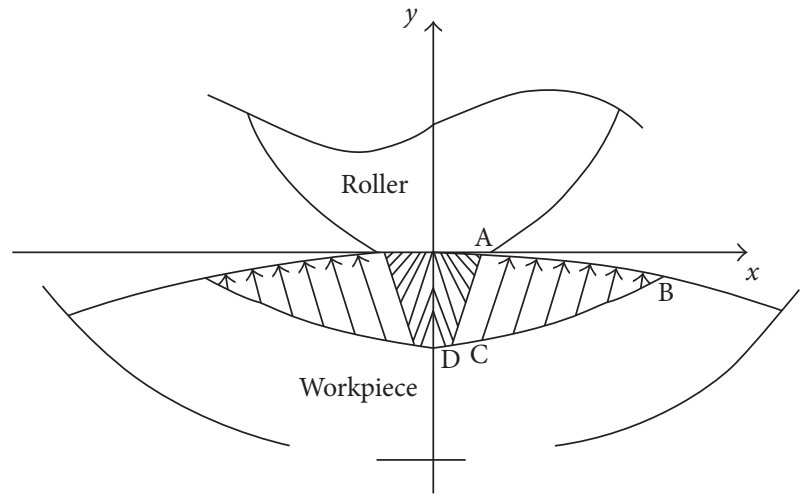

FIgURE 1: Contact model of cold rolling.

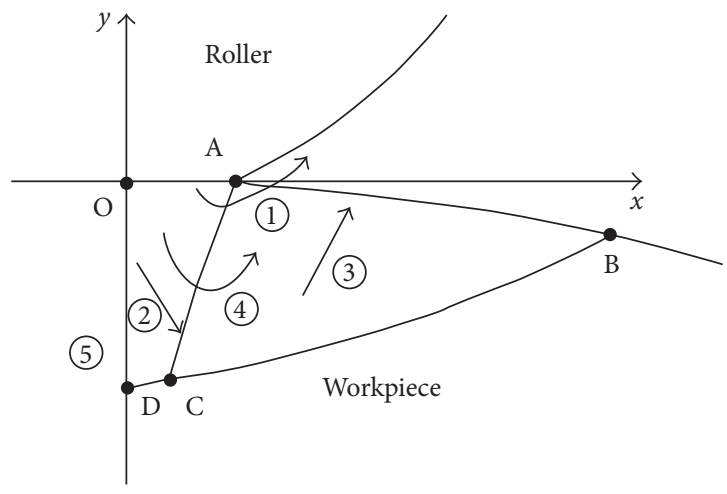

Figure 2: Analytic result of metal flowing trend.

paper regarding the metal plastic flow law. By extracting the residual stress from the surface of the spline tooth profile, this paper clarified the effect of the metal plastic flow process on the residual stress formation in the profile of spline teeth. At the end, the forming mechanism of the residual stress under high-speed cold roll-beating was revealed, and the experiment verified the theoretical and finite element simulation parts of this paper.

\section{Metal Flowing and Residual Stress Forming Mechanism of the Complex Functional Profile under High-Speed Cold Roll-Beating}

2.1. Metal Flowing of the Complex Functional Profile under Cold Roll-Beating. By analyzing the process of cold rollbeating, Cui et al. [19] established the contact model of a roller and a workpiece, as shown in Figure 1. The trend of metal flow at the involute spline tooth profile is determined by the law of least resistance, as shown in Figure 2.

It can be seen from the metal flow trends shown in Figure 2 that with the invasion of the roller, the metals in the $\mathrm{ACDO}$ region gradually flow into the $\mathrm{ABC}$ region and the region outside the $\mathrm{AB}$ border. The new inflowing metals force the metal in the $A B C$ region to flow to the $A B$ boundary. In the flowing process, because the metals near the top position of the roller (especially near the $\mathrm{C}$ point on the $\mathrm{CD}$ online) flow under the direct compression force of the roller, the flow rate is the largest. As a result, the most 


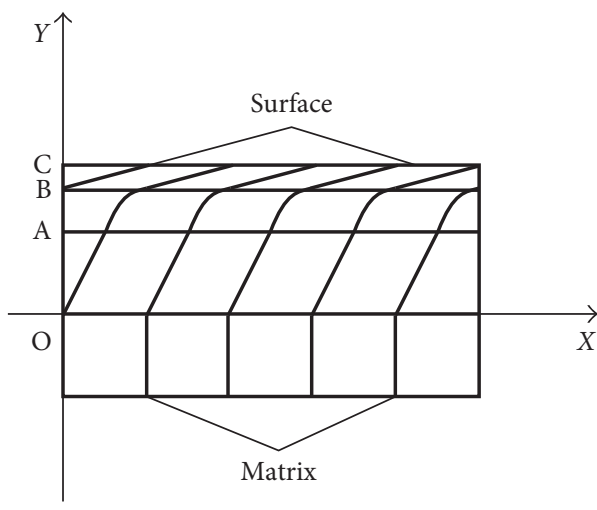

(a)

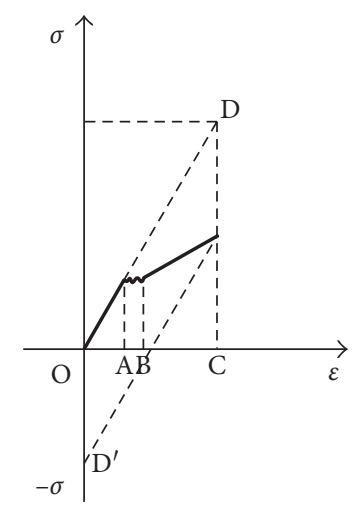

(b)

Figure 3: Schematic form of residual stress. (a) Metal flowing. (b) Generation model of residual stress.

uneven plastic flow occurred in this region. The CA line, due to the metals near point $\mathrm{C}$, suffered much greater rolling stress than did the area near point $\mathrm{A}$, and the resistance of the metal flow in the $\mathrm{ABC}$ area is greater than that to the flow along the line CA. Therefore, when deformation occurs, parts of the metals at $C$ point in the $A C D O$ area flow into the $A B C$ region, and other parts flow along the CA line direction. However, close to point A, the flow pattern weakens gradually. Therefore, the degree of unevenness on the metal plastic flow gradually decreases along the CA line direction, as does the residual stress. However, in the $\mathrm{ABC}$ region, since the metal is subjected to indirect forces of the roller from flowing, the force and metal plastic flow are relatively uniform.

\subsection{Residual Stress Forming of the Complex Functional Profile} under High-Speed Cold Roll-Beating. It is known from the above analysis that due to the nature of the metal material and the effect of the changing force during the cold rollbeating forming process, the metallic material flow of the workpiece cannot be uniform, leading to the interaction of the plastic deformation portion and the elastic deformation portion inside the workpiece structure. Finally, an elastic stress field is formed. Under the dual action of the elastic stress field recovery and plastic deformation hindrance, the internal residual stress is produced. The formation diagram is shown in Figure 3. The metal flowing at different depth layers during the forming process is described in Figure 3(a), and the generation model of residual stress is described in Figure 3(b).

In Figure 3(a), the $O X$ axis represents the flow direction of the metal surface layer, and the $O Y$ axis represents the thickness of the workpiece metal layer. The OA segment is the elastic deformation area. The $\mathrm{AB}$ segment shows the elastic-plastic deformation zone. The $\mathrm{BC}$ section is the plastic deformation zone. In Figure 3(b), the $O \varepsilon$ and $O \sigma$ axes indicate the workpiece material strain and stress, respectively. $\mathrm{OA}, \mathrm{AB}$, and $\mathrm{BC}$ represent the elastic deformation stage, yield stage, and effective plastic strain-strengthening stage, respectively. Without considering the plastic deformation of the material, point $\mathrm{D}$ is the maximum stress that the material can achieve before reaching the material's strain limit. Point $\mathrm{D}^{\prime}$ is the residual stress produced by the ideal elastic recovery action after the external force is removed.

The schematic of the residual stress formed is shown in Figure 3(a). When the metal plastic flow occurs on the surface layer of the workpiece, the deformation resistance of the matrix layer (the sections below the $O X$ axis) is greater, but hardly any deformation appears. However, the elastic deformation gradually increases in zone OA, which is far from the matrix layer and near the surface layer. Then, some metals yield and enter the transition area $\mathrm{AB}$ of elastic-plastic deformation. After the external force is removed, the elastic deformation has a tendency to recover but is still affected by plastic deformation in the surface area; the matrix area and the existing state of elastic deformation will be maintained. The stored deformation can be expressed as residual stress.

The value of the residual stress formed is shown in Figure 3(b). Workpiece materials under the action of the external force first produce an elastic deformation (OA). With an increase in external force, workpiece materials enter the yield stage $(\mathrm{AB})$. As the increases continue, materials enter the effective strain-strengthening phase (BC). If the plastic deformation has not occurred and the strain reaches point $C$, the material stress reaches point $D$, and the stress and strain return to the origin after removing the external force. In fact, because of the plastic deformation of the materials and the deformation field recovering along the dotted line, in parallel with the elastic line, strain still exists in the material when the stress has decreased to zero. Hence, to restore the strain to zero, the stress values should change continually along the dotted line until reaching the intersection with the negative side of the longitudinal axis, as shown at point $\mathrm{D}^{\prime}$ in Figure 3(b). The stress value at point $\mathrm{D}^{\prime}$ is the size of the residual stress value.

\section{Simulation Analysis of Metal Flow and Residual Stress Forming of a Complex Functional Profile under Cold Roll-Beating}

Taking the involute spline for high-speed cold roll-beating forming as an example and using finite element simulation 
TABLE 1: J-C material model parameters.

\begin{tabular}{lcccccc}
\hline$A(\mathrm{MPa})$ & $B(\mathrm{MPa})$ & $C$ & $m$ & $n$ & $E(\mathrm{MPa})$ & $\mu$ \\
\hline 213 & 53 & 0.051 & 0.76 & 0.345 & $2.13 e 5$ & 0.282 \\
\hline
\end{tabular}

TABLE 2: Other physical parameters.

\begin{tabular}{lcccccc}
\hline $\begin{array}{l}E \\
(\mathrm{MPa})\end{array}$ & $\mu$ & $T_{\mathrm{r}}(\mathrm{K})$ & $T_{\mathrm{m}}(\mathrm{K})$ & $\begin{array}{c}K \\
(\mathrm{~W} /(\mathrm{m} \cdot \mathrm{K}))\end{array}$ & $\begin{array}{c}\mathrm{SPH} \\
(J /(\mathrm{kg} \cdot \mathrm{K}))\end{array}$ & $\alpha$ \\
\hline $2.13 e 5$ & 0.286 & 1623 & 293 & 48.00 & 440 & $1.19 e-5$ \\
\hline
\end{tabular}

software, the metal flow conditions and residual stress on the tooth profile during the forming process were simulated. A schematic of the cold roll-beating forming spline was established by Cui $[13,19]$.

\subsection{Model Building}

3.1.1. Finite Element Model. According to the cold rollbeating forming movement process given in [13] and [19], the roll-beating model is simplified by analyzing half of the symmetric model. To improve the efficiency of the analysis without affecting the results, the workpiece's selfrotation was ignored, and a single tooth profile surface formed under roll-beating was analyzed. The axial dimension of the semi-finished shaft is set as $60 \mathrm{~mm}$, and the semi-finished shaft diameter is $35.15 \mathrm{~mm}$, as obtained by the estimating equation. According to the standard inverse involute equation, the tooth profile of the roller is established; its radius is $19 \mathrm{~mm}$, thickness is $8 \mathrm{~mm}$, modulus is 2.5 , and rounded value is $0.5 \mathrm{~mm}$. The gyration radius is $55 \mathrm{~mm}$, and the strike depth is $1.95 \mathrm{~mm}$.

Since the roller partially contacts the workpiece during the roll-beating forming process, the workpiece mesh block, and the partial contact mesh refinement, the element type selected is the Solid 164 entity structure unit.

3.1.2. Material Model. Through dynamic compression tests on 1020 steel with the cold roll-beating experimental platform, the stress-strain data were obtained at different temperatures and strain rates. The $\mathrm{J}-\mathrm{C}$ material model parameters were established by the data, listed in Table 1, and other physical parameters, listed in Table 2.

Relative to the workpiece material (1020 steel), the deformation of the roller with a material of $\mathrm{Cr} 12 \mathrm{MoV}$ does not need to be considered in the high-speed cold roll-beating forming process. In this situation, the roller is defined as rigid, according to the movement of the roller. The rotational freedom along the $Z$-axis is retained only with the definition of the rigid material model.

3.2. Key Simulation Parameters. The symmetry constraint is applied on the symmetry plane of the workpiece, and displacement constraints are applied on the rear and perpendicular to the workpiece feed direction. A surface-to-surface eroding contact is used between the roller and the spline

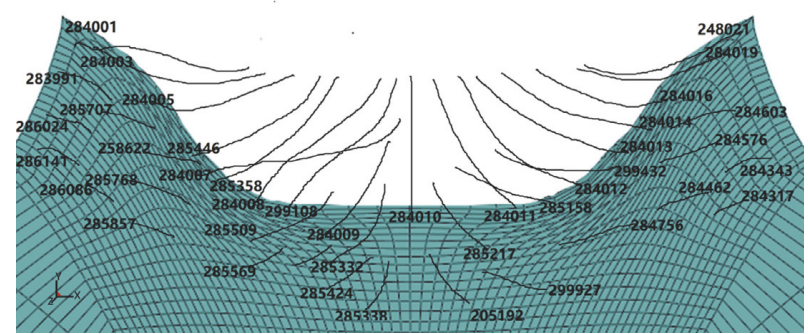

FIgURE 4: Metal flow trajectory in the simulation.

shaft blank. The dynamic and static friction coefficients are set as 0.12 and 0.2 , respectively. The hourglass control and volume viscosity are set as the system defaults. The feed rate of the workpiece is $0.5 \mathrm{~mm} / \mathrm{s}$, and different rotational speeds of the roller are set as $188.5 \mathrm{rad} / \mathrm{s} \quad(1800 \mathrm{r} / \mathrm{min})$ and $235.6 \mathrm{rad} / \mathrm{s}(2250 \mathrm{r} / \mathrm{min})$ for comparison.

\subsection{Simulation Results and Analysis}

3.3.1. Metal Flowing Analysis. The metal flow patterns obtained by simulation under different roll-beating speeds are very similar, so the metal flow trajectory under the $188.5 \mathrm{rad} / \mathrm{s}$ speed is depicted in Figure 4.

As seen in Figure 4, the contact area near the peripheral area of the node (e.g., nodes 284019, 284016, and 284014) flows to the blank area in the upper right. The flow path can be attributed to the number 1 trace line in Figure 2. In the area near the center line, the main flow direction of the node (e.g., nodes 285217 and 285192) is obliquely downward, with an angle toward the center line. The flow trajectory is nearly linear and can be attributed to the number 2 trace line in Figure 2. The nodes on both sides (e.g., nodes 284603, $284576,284462,284343$, and 284317) that are far from the deformation zone flow to the region outside the shaft blank in a certain direction and along an approximately linear trajectory that can be attributed to the number 3 trace line in Figure 2. The node (e.g., nodes 299432 and 285158) is located in the middle of the right half of the gullet flow in the forming region and has difficulty flowing to the region outside the shaft blank. The flow trajectory is a certain curve and can be attributed to the number 4 track line in Figure 2. The nodes represented by node 284010 on the center line flow down along the center line of the cogging that are summed in Figure 2 on the number 5 track line.

Comparing the simulation results of the metal overall flow trend (Figure 4) and the theoretical analysis of the metal flow trend (Figure 2), the conclusions are consistent.

3.3.2. Residual Stress Analysis. The main failure modes of spline teeth are fatigue fracture and tooth surface pitting; fatigue fractures occur at the root of the tooth, and the dedendum is exactly the stress concentration point. In the case of continuous stress shocks, fatigue cracks are the most likely to occur, resulting in broken dedendum. Residual compressive stress will inhibit the generation and propagation of cracks, thereby increasing the fatigue strength at 


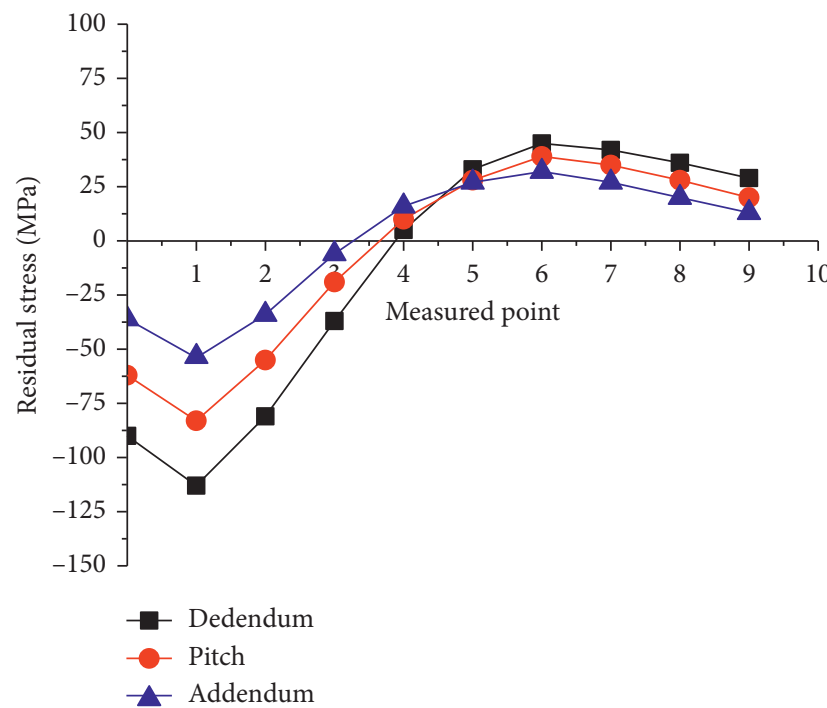

(a)

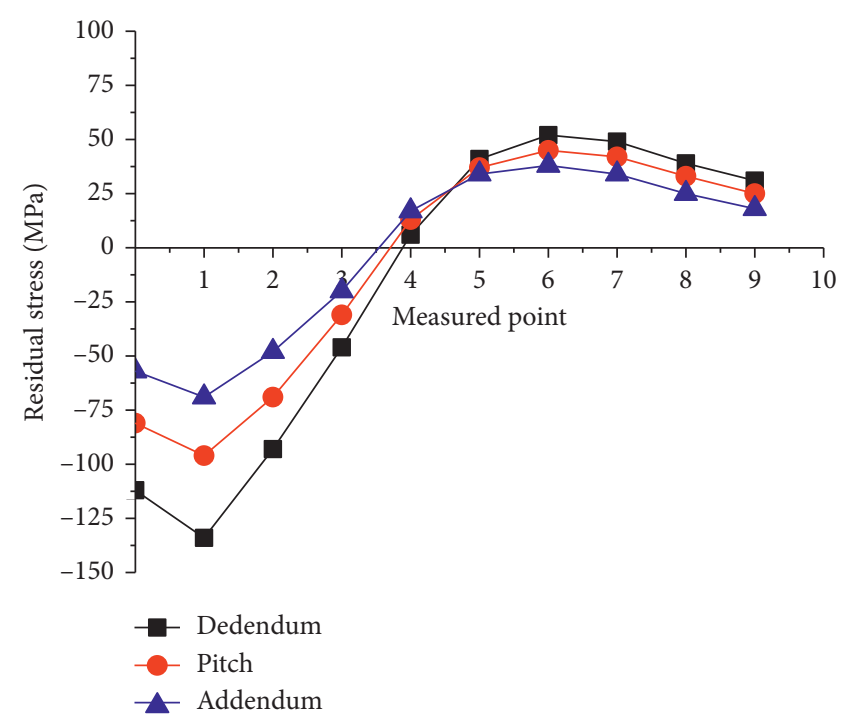

(b)

Figure 5: Residual stress distribution curve under different rotation speeds: (a) $188.5 \mathrm{rad} / \mathrm{s}$ and (b) $235.6 \mathrm{rad} / \mathrm{s}$.

the tooth roots. Therefore, the use of splined dedendum is selected as a research point. The tooth surface pitting occurs mostly at the pitch of the splines, and the main contact portion of the two spline teeth in the meshing process is the pitch; in the meshing process, spline teeth have both relative rolling and relative sliding, and the relative sliding friction force is opposite in direction of the two sides of the pitch circle node; therefore, a pulsating load is generated, and the effect of these two forces results in pulsating cyclically varying shear stress at the depth of the gear index circle. When the shear stress exceeds the shear fatigue limit of the gear material, the surface will generate fatigue cracks, and the crack propagation will eventually cause the tooth surface to flake off and form a small crater on the tooth surface; therefore, it is selected as the research point to select the pitch of spline. During the cold roll forming process, the splined addendum is generated by the metal flow, so the splined addendum is selected as the research point, and the fatigue strength at the tooth tip is mainly checked by measuring the residual stress.

The internal residual stress distribution curves for dedendum, pitch, and addendum of the spline tooth profile under different speeds are shown in Figure 5.

As seen in Figure 5, the residual stress distribution curve trends of tooth profiles of three positions under the two different speeds are roughly the same. However, as the speed increases, there is an increasing trend of residual stress on the whole tooth profile. The residual stress distribution curve from different locations on tooth profiles at the same speed shows that the surface of the tooth profile is compressive residual stress. With a measurement depth to the tooth surface (measured point increasing), the internal tooth profile gradually shows the tensile residual stress, and the maximum value and gradient of the compressive stress are larger than those of the tensile residual stress. Residual stress reaches the maximum at the dedendum and the minimum at the addendum.

Combined with the theoretical section of this paper, as the roll-beating speed increases, the strain rate of the workpiece increases, leading to an increase in the material flow stress in the deformation. Thereby, the deformation depth of the workpiece layer is affected; however, because the deformation resistance of metal at depth is larger than that at the surface, deformation does not occur easily at depth, and the plastic flow on the surface of workpiece is more uneven. Therefore, as the rotating speed increases, the residual stress on the whole tooth profile also has an increasing trend. In the root of the spline tooth profile part, because of the direct contact with the outer edge of the roller, the strain, strain rate, and deformation resistance are larger than those at the pitch and addendum position during deformation. As a result, the degree of unevenness of the metal flow increases, and the final residual stress formed here is the largest. In the addendum position, the force of the workpiece material caused by the roller is reduced, and the metal's flow decreases. Residual stress internal to the metal is gradually released at the same time; therefore, the residual stress is less than that at the pitch position.

\section{Experimental Study of Metal Flow and Residual Stress Formation}

4.1. Experimental Scheme. The 1020 steel blanks come from the same batch. The samples are machined by a Swiss ZRMe9 roll-beating machine from Grob Inc. The modulus of the involute spline is 2.5 . There are 14 teeth. The forming method is pulling out down-beating and continuous indexing. The samples are divided into two groups. The processing parameters of the first group are as follows: the spindle speed is $1809 \mathrm{r} / \mathrm{min}$ and the feed rate is 


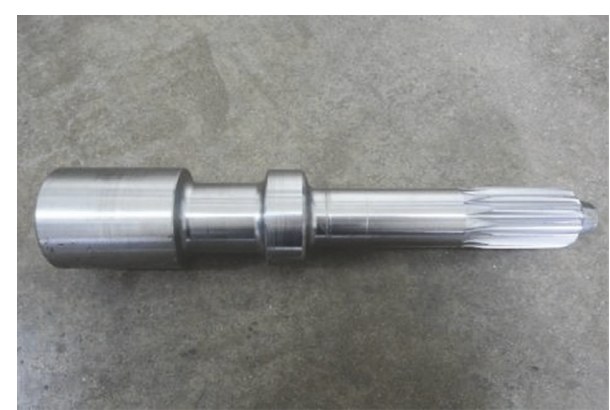

Figure 6: Processed involute spline of cold roll-beating.

$28 \mathrm{~mm} / \mathrm{min}$. The parameters of the second group are as follows: the spindle speed is $2258 \mathrm{r} / \mathrm{min}$ and the feed rate is $28 \mathrm{~mm} / \mathrm{min}$. The involute spline after processing is shown in Figure 6.

A tooth of the spline was cut by wire electric discharge machining (wire EDM). The surface of the tooth section was polished, and it was cut from the individual tooth along the tooth direction. After cleaning the surface with alcohol swabs and rinsing the surface with alcohol containing $4 \%$ concentrations of nitric acid for 50 seconds (until the surface of the sample was light brown), the samples were finally obtained.

Using a JSM-5610LV scanning electron microscope (SEM), the microstructures and morphologies at the position of the tooth top, the dividing circle, and the dedendum of the tooth section in two groups were observed. The electron acceleration voltage was $20 \mathrm{kV}$.

In the WEDM-LS procedure, using a $0.1 \mathrm{~mm}$ bronze wire, a tooth is cut off from the spline at a speed of $2 \mathrm{~mm} / \mathrm{min}$, and the specimen is then cut along the symmetrical surface shown in the shaded part of Figure 7 (a) at a feed rate of $0.5 \mathrm{~mm} / \mathrm{min}$. The dimensions of each specimen are as follows: $l=10 \mathrm{~mm}, w=4.35 \mathrm{~mm}$, and $h_{0}=2.68 \mathrm{~mm}$. The point coordinates of the cutting plane of Figure 7 (a) were measured using a Serein-CMM FUNCTION 1000 three-coordinate measuring machine (to reduce the error, both surfaces produced by cutting must be measured, for a total of four surfaces). Measurements were performed at $0.01 \times 0.01 \mathrm{~mm}$ intervals using the reciprocating measurement method (a single measurement track along the direction parallel to the cutting line is required to cover the two surfaces). The annealed samples are considered not to contain residual stress, so after the cutting, any form of deformation of the unannealed specimen relative to the corresponding position of the annealed specimen can be attributed to the release of residual stress. After measurement, the measured data that correspond to the two planes are subtracted to obtain the measured point change amount (vector deformation), which is the amount of deformation caused by the release of the residual stress of the specimen. A curved surface for the small change (annealed specimen relative to the unannealed one) corresponding to each measured point is fitted by using three order spline fitting algorithm. Then, the surface was inverted and taken as a boundary condition to be applied in the finite element model, with the same size and shape as the deformed specimen, using Abaqus software. The material model used in the simulation analysis was a static stress-strain simulation that was established using quasistatic compression tests on 20 steel samples on the experimental platform. Then, a finite element static solution was performed in the Abaqus software, and the deformed model was restored to the shape before cutting. To avoid rigid body displacement in the model analysis process, an additional constraint that did not affect the free deformation of the contour was imposed on the corner node at the other end of the model, consistent with the literature [20-23]. Finally, the stress on the cut surface of the model obtained after the solution was obtained was equivalent to the residual stress at the same position when the sample was not cut. The extraction direction and position are shown in Figure 7(b). The residual stress at the spline circle is extracted by the position and direction of $b$, and the extraction node spacing is approximately $0.05 \mathrm{~mm}$; some distortion exists, so it is not possible to select nodes with strict regular spacing. In this case, $h_{\mathrm{a}}=2.20 \mathrm{~mm}$, $h_{\mathrm{d}}=1.39 \mathrm{~mm}$, and $h_{\mathrm{f}}=0.5 \mathrm{~mm}$.

\subsection{Metal Plastic Flow Analysis of the Experimental Results.} The microstructures of the spline tooth section in the two experimental groups are shown in Figures $8(\mathrm{a})$ and $8(\mathrm{~b})$, respectively. In the images, from top to bottom, the position of addendum, pitch, and dedendum are at the distances of $0.1 \mathrm{~mm}, 0.5 \mathrm{~mm}$, and $0.9 \mathrm{~mm}$, from left to right.

Compared with the two groups' microstructure morphology results of splines obtained from the experiments, the metal grains change significantly at different speeds (different strain rates). At a higher strain rate, a larger amount of grain deformation occurs inside the metal, and a higher degree of unevenness is produced. According to the direct invasion influence of the roller on the spline surface, metals flow rapidly along the roller surface, and a dense fibrous structure is formed. Since the internal metals of the spline teeth suffer an indirect force caused by the surface, the stress is relatively uniform, and the resistance of the movement is larger in this part than it is on the surface. Therefore, along the surface to the internal area, the elongated degree of the grain is reduced, and the metal plastic flows weaken gradually.

The microstructure topographies at three different positions of two spline sectionals show that, at the same rolling speed, the elongated degree of the grain in different positions of the spline cross section is also different. The nonuniform degree of grain is highest in the tooth position, and grains are stretched significantly. The elongation at the addendum is not as high, and the grain deformation is similar. The degree of grain deformation at the pitch position is between those at the dedendum and addendum. Comparing the grain deformation in different locations, the degree of nonuniform metal plastic flow is the highest in the root position, followed by the pitch, and the flow is the lowest at the addendum.

In summary, a larger strain rate leads to a larger nonuniform degree of metal plastic flow. At the same strain rate, 


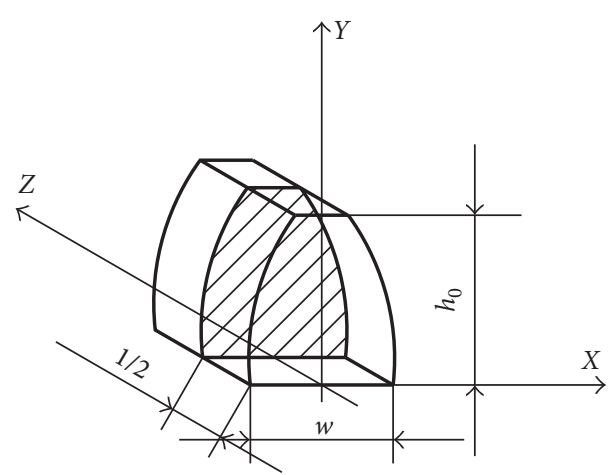

(a)

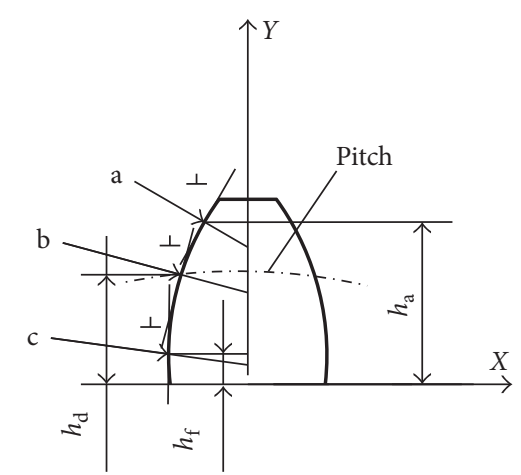

(b)

Figure 7: Measured scheme of residual stress of the contour method. (a) Sample shape and cutting position needed for measurement. (b) Measured position and direction.

the degree of incomplete plastic deformation is the highest in the root position, followed by the graduated circle, and is the lowest in the addendum position. This is entirely consistent with the previous analysis.

4.3. Residual Stress Analysis of the Experimental Results. The residual stresses in the root, pitch, and addendum, as measured by the contour method at $1809 \mathrm{r} / \mathrm{min}$ and $2258 \mathrm{r} / \mathrm{min}$ spline speeds, are shown in Figures 9(a) and 9(b), respectively. Only the residual stress on the section from the left tooth surface to the $Y Z$ plane symmetry is depicted because the single spline tooth is symmetrical with respect to the $Y Z$ plane. The distances of three positions on the left flank to center symmetry are $2.14 \mathrm{~mm}, 2.00 \mathrm{~mm}$, and $1.82 \mathrm{~mm}$. The stress measurement point is located on the sample $1 / 2$ symmetry plane with a $0.25 \mathrm{~mm}$ interval measurement to the $Y Z$ plane.

Compared with the residual stress size and distribution shown in Figures 9 and 5, the experimental and simulation results have a high degree of consistency in the overall curve trend. These results show that compressive residual stress is formed on the surface of the tooth profile and that tensile residual stress is formed deep below the surface. The residual stress is larger at the dedendum than are those at the pitch and the addendum, and it is smallest at the addendum. Compared with the results obtained at the two forming speeds, the residual stress tends to increase as the spindle speed increases. These results all coincide with the theory and simulation.

The sizes of the residual stress obtained by experiments and simulations show that the residual stress from the numerical simulation is slightly larger in the whole system. High-speed cold roll-beating is a formation process with large deformation, a high strain rate, a high instantaneous temperature, and high stress values. The process may lead to transformation in the area of the workpiece and cause the residual stress to change. Furthermore, after forming, residual stress on spline shaft tooth profile surface will gradually release and decrease over time. However, in the cold roll-beating forming simulation, to ensure that the model is not too complex, the analysis failed to take the transformation and residual stress releasing gradually over time during the forming process into account (as the time increases, the releasing roughly obeys a logarithmic curve form). Thus, the final residual stress values that are from the experiments are slightly lower than those of the numerical simulation.

\section{Discussion}

Compared with the simulation (Figure 5) and experimental (Figure 9) residual stress results, at high spindle speeds, the value and depth of residual stress are both larger because the nature of the residual stress is generated by the nonuniform plastic deformation of the workpiece material. At a high spindle speed, an increase in the workpiece material strain rate causes a greater material flow stress, greater effect depth in the workpiece, and higher degree of inhomogeneous deformation of the workpiece material. As a result, the depth and value of residual stress increase finally. At a high spindle speed, the increase in the inhomogeneous deformation of the workpiece material could be determined by the experiment (Figure 8). During the forming process, the grains on the tooth profile are stretched, and the lattice is distorted. The elongated degree of the grains on the tooth profile of the workpiece and the depth of tensile deformation grains are both greater at high spindle speeds.

The residual stress distribution is different at different positions of the tooth profile during the high-speed cold rollbeating forming process. As seen from the simulation (Figure 4) and the experimental metal flow (Figure 8), the degree of metal plastic deformation caused by the metal flow is different in different parts of the tooth profile. The degree of metal plastic deformation is clearly the largest in fillet parts of the dedendum. In this position, the severe deformation element extrudes deeper into the element in the simulation. The grain is pulled into a dense fiber whose thickness is larger than elsewhere under a scanning electron microscope. The metal plastic deformation degree decreased at the position of the pitch circle and is smallest at the addendum. 

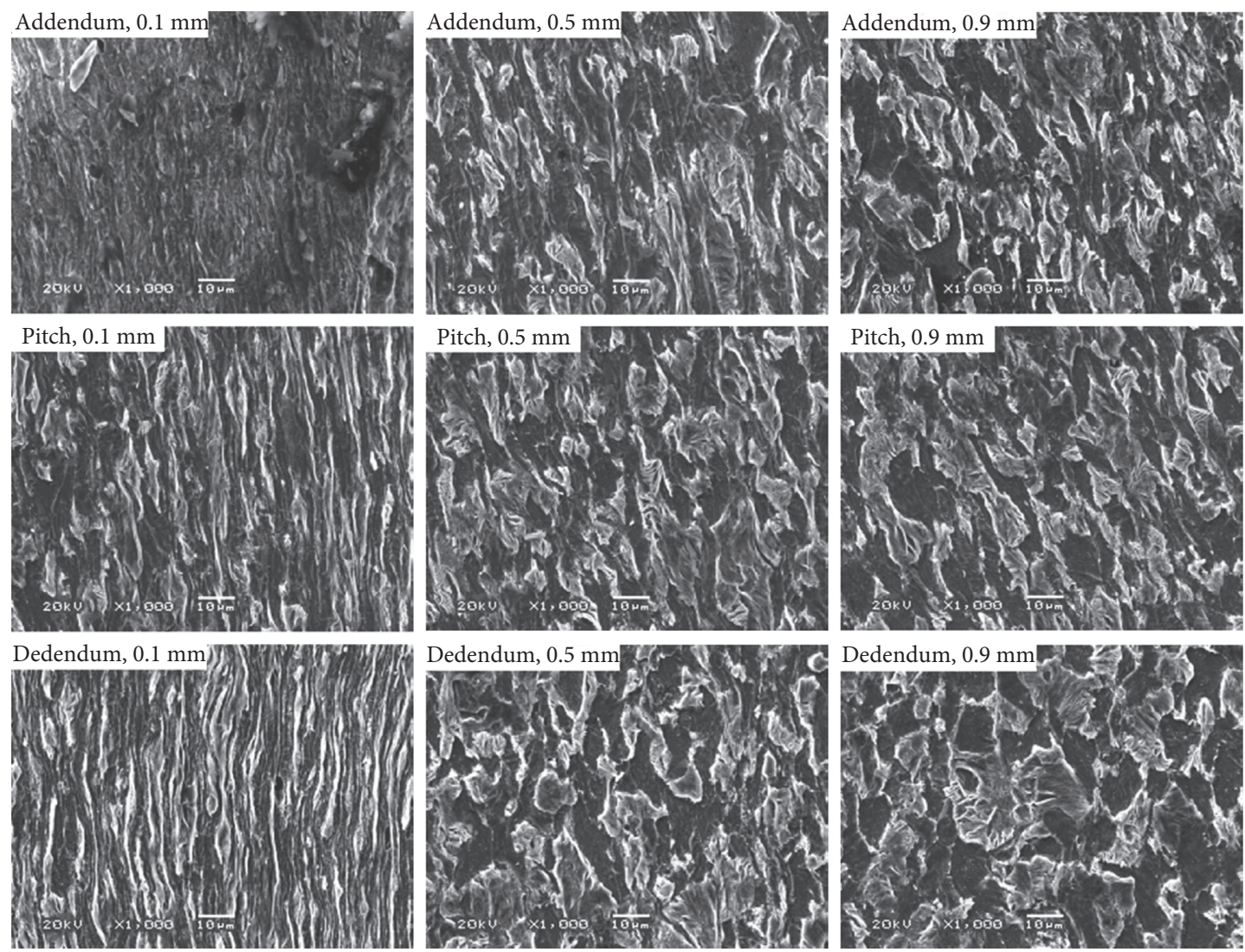

(a)
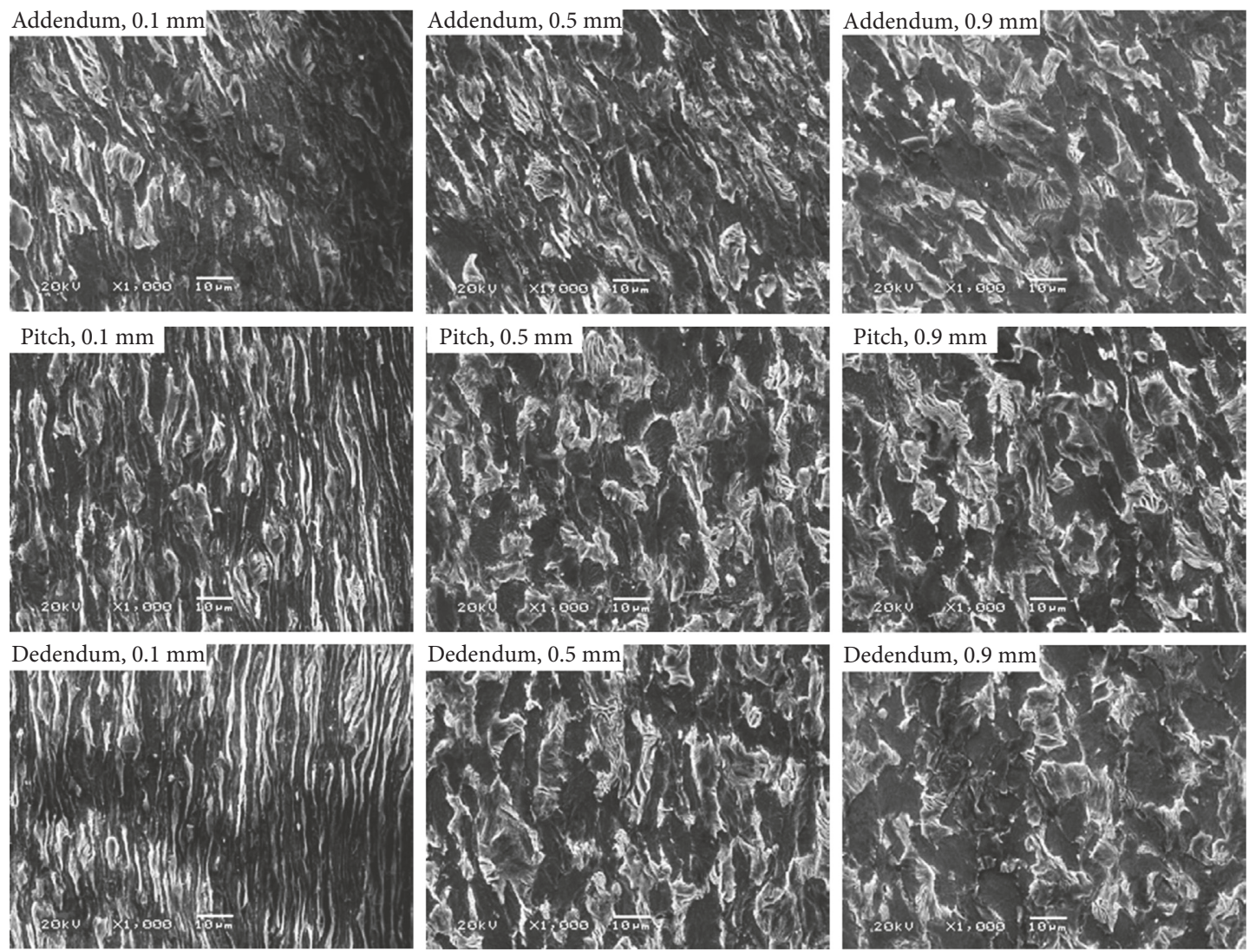

(b)

FIGURE 8: Microstructure morphology under different spindle rotation speeds: (a) $1809 \mathrm{r} / \mathrm{min}$ and (b) $2258 \mathrm{r} / \mathrm{min}$. 


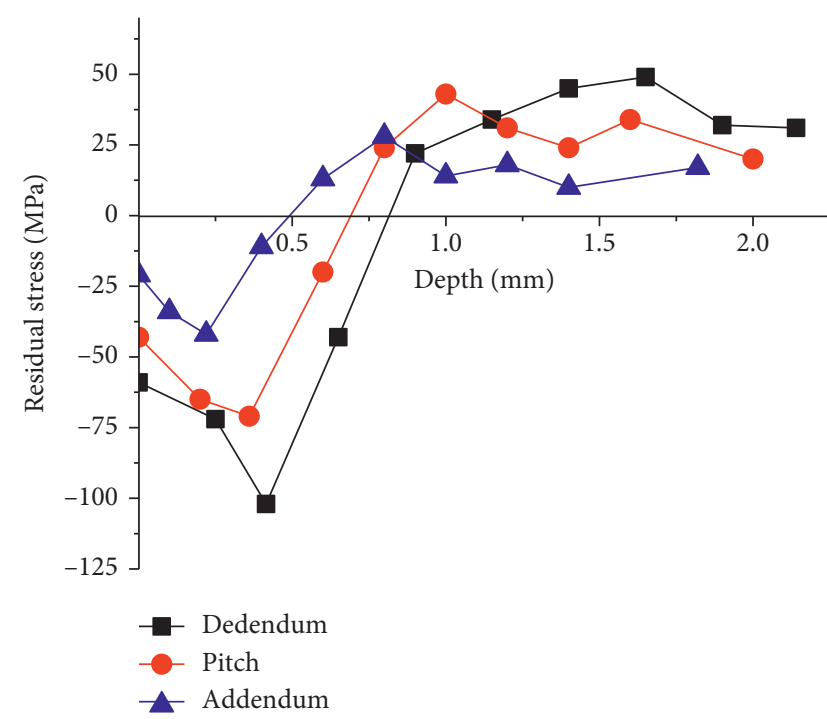

(a)

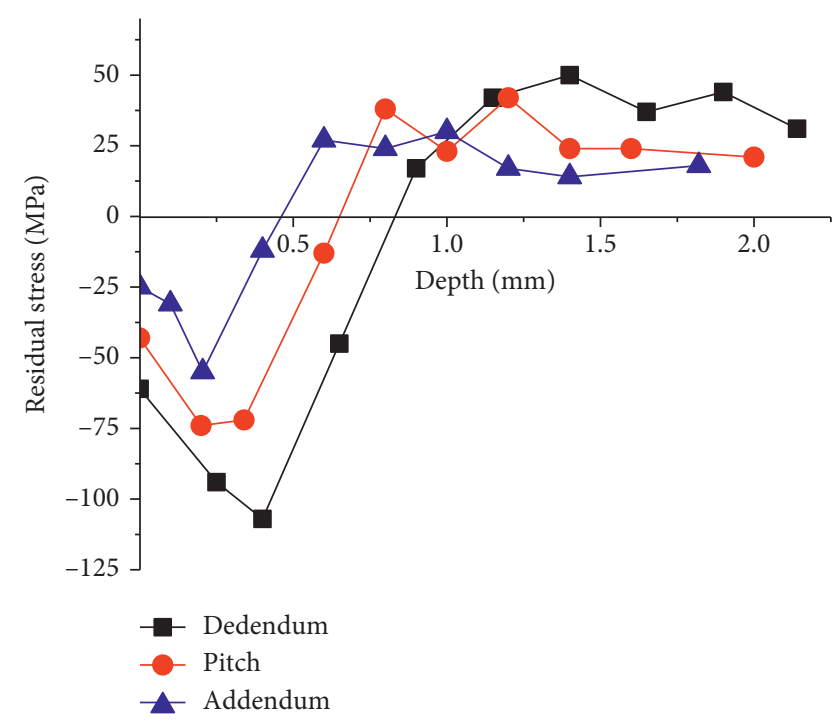

(b)

FIgURe 9: Distribution and size of the residual stress at three locations under two spindle speeds: (a) $1809 \mathrm{r} / \mathrm{min}$ and (b) $2258 \mathrm{r} / \mathrm{min}$.

In summary, the residual stress of a cold roll-beating spline shaft occurs mainly because of the nonuniform plastic deformation caused by the plastic flow of the local metal in the workpiece that is extruded by the roller. In the residual stress formation process, the workpiece surface metal has the largest extrusion force and flow rate, which both decrease inwardly with a larger decreasing gradient. A large degree of uneven metal plastic deformation is produced, and residual compressive stress is formed on the workpiece's surface. Residual tensile stress is formed in the workpiece's interior because of the mutual balancing effect between the workpiece interior and the surface. Affected by the shape of the roller, the metal flow of the tooth profile is different. The degrees of metal plastic flow and uneven plastic deformation are the highest at the dedendum, followed by the pitch circle, and are the smallest at the tooth top. The largest residual stress is ultimately formed at the dedendum circle, followed by the pitch circle, and the smallest is found at the addendum.

\section{Conclusion}

(1) In the cold roll-beating process, the metal plastic flow rate is different between the surface and inner areas of the location. The metal flow rate of the surface is largest, and it hardly occurs at the base layer. The decreasing rate of the metal flow is gradually reduced.

(2) The flow deviation is caused by the changes in the metal flow rate. It leads to plastic deformation nonuniformity and forms the metal residual stress. The residual stress value is directly related to an uneven plastic flow.

(3) At a high roll-beating speed, the degree of plastic deformation at the workpiece surface is more uneven, and the residual stress in the tooth profile is larger overall.
(4) At the same roll-beating speed, the rate change trend of the metal flow gradually decreases along the direction of the surface to the inner layer from the dedendum to the addendum and is the highest at the fillet parts of the dedendum. The residual stress distribution on the surface of the tooth profile decreases from the dedendum to the addendum.

\section{Conflicts of Interest}

The authors declare that they have no conflicts of interest.

\section{Acknowledgments}

This project was sponsored by the National Natural Science Foundation of China (Grant nos. 51475146, 51475366, and 51075124).

\section{References}

[1] P. A. F. Martins, N. Bay, M. Skjoedt, and M. B. Silva, "Theory of single point incremental forming," CIRP AnnalsManufacturing Technology, vol. 5, no. 57, pp. 247-252, 2008.

[2] M. B. Silva, M. Skjoedt, A. G. Atkins, N. Bay, and P. A. F. Martins, "Single-point incremental forming and formability-failure diagrams," Journal of Strain Analysis for Engineering Design, vol. 1, no. 43, pp. 15-35, 2008.

[3] E. M. Viatkina, W. A. M. Brekelmans, and M. G. D. Geers, "Modelling of the internal stress in dislocation cell structures," European Journal Mechanics-A/Solids, vol. 5, no. 26, pp. 982-998, 2007.

[4] M. S. Abdul Aziz, T. Furumoto, K. Kuriyama et al., "Residual stress and deformation of consolidated structure obtained by layered manufacturing process," Journal of Advanced Mechanical Design System and Manufacturing, vol. 2, no. 7, pp. 244-256, 2013. 
[5] L. Del Llano-Vizcaya, C. Rubio-Gonzalez, G. Mesmacque, and A. Banderas-Hemandez, "Stress relief effect on fatigue and relaxation of compression springs," Materials and Design, vol. 4, no. 28, pp. 1130-1134, 2006.

[6] T. Furumoto, T. Ueda, M. S. A. Aziz, A. Hosokawa, and R. Tanaka, "Study on reduction of residual stress induced during rapid tooling process: influence of heating conditions on residual stress," Key Engineering Materials, vol. 9, no. 447448, pp. 785-789, 2010.

[7] C. J. Lammi and D. A. Lados, "Effects of residual stresses on fatigue crack growth behavior of structural materials: analytical corrections," International Journal of Fatigue, vol. 2, no. 33, pp. 858-867, 2011.

[8] F. K. Cui, W. Zhao, Y. F. Xu, and J. X. Yang, "Research on fibrous structure and surface work hardening test of cold rolling involute spline," Machine Tool and Hydraulics, vol. 3, no. 37 , pp. $36-38,2010$.

[9] F. K. Cui, X. D. Dong, X. Q. Wang et al., "The analysis of macroscopic property changes and microstructural evolution of 1020 steel under cold beating," Materials Research Innovations, vol. 4, no. 19, pp. 56-61, 2015.

[10] H. Mizutani and M. Wakabayashi, "Influence of cutting edge shape on residual stresses of cut surface (effects of nose radius and cutting edge roundness)," Journal of Advanced Mechanical Design System and Manufacturing, vol. 4, no. 14, pp. 1201-1209, 2008.

[11] G. A. Smirnov, Study of Finite Plastic Deformation in Cold Form Rolling of Splines on Shafts, Macmillan, London, 1971.

[12] N. Kurz, "Theoretical and experimental investigations of the 'grob' cold shape-rolling process," in Proceedings of the Twenty-Fifth International Machine Tool Design and Research Conference, pp. 551-559, Birmingham, UK, April 1985.

[13] F. K. Cui, Study of High-Speed Precise Forming with Cold RollBeating Technique, Xi'an University of Technology, Xi'an, China, 2007.

[14] L. Zhang, M. S. Yang, Y. Li, and Q. L. Yuan, "Analytic method and its modification for deformation force of high-speed cold roll-beating forming," Journal of Plasticity Engineering, vol. 3, no. 18, pp. 1-7, 2012.

[15] E. Grob and H. Krapfenbauer, "Roller head for cold rolling of splined shafts or gears," US Patent 3818735A, 1973.

[16] M. Weck, W. Koenig, G. Bartsch, and K. Steffens, "Manufacture and load-bearing capacity of cold-rolled gears," in Proceedings of 3rd International Conference on Rotary Metalworking Processes, pp. 395-406, Kyoto, Japan, September 1984.

[17] F. K. Cui, K. G. Xie, Y. F. Xie, X. Q. Wang, W. J. Zhu, and Y. Li, "Analysis of coupled thermal-mechanical mechanism based on work hardening phenomenon in high-speed cold rollbeating," Materials Research Innovations, vol. 5, no. 19, pp. 1212-1218, 2015.

[18] F. K. Cui, Y. F. Xu, and W. Zhao, "Research on metal microstructure deformation of splines manufactured by cold rolling, milling and cutting processes," Forging and Stamping Technology, vol. 7, no. 32, pp. 70-74, 2008.

[19] F. K. Cui, Y. F. Xie, X. D. Dong, and L. M. Hou, "Simulation analysis of metal flow in high-speed cold roll-beating," Journal of Henan Polytechnic University, vol. 9, no. 33, pp. 467-471, 2014.

[20] M. B. Prime, "Cross-sectional mapping of residual stresses by measuring the surface contour after a cut," Journal of Engineering Materials and Technology, vol. 4, no. 123, pp. 162-168, 2001.
[21] P. Pagliaro, M. B. Prime, J. S. Robinson et al., "Measuring inaccessible residual stresses using multiple methods and superposition," Experimental Mechanics, vol. 9, no. 51, pp. 1123-1134, 2011.

[22] P. Pagliaro, M. B. Prime, H. Swenson, and B. Zuccarello, "Measuring multiple residual-stress components using the contour method and multiple cuts," Experimental Mechanics, vol. 50, no. 2, pp. 187-194, 2010.

[23] P. Pagliaro, M. B. Prime, M. L. Lovato, and B. Zuccarello, "Known residual stress specimens using opposed indentation," Journal of Engineering Materials and Technology, vol. 131, no. 3, pp. 0310021-03100210, 2009. 


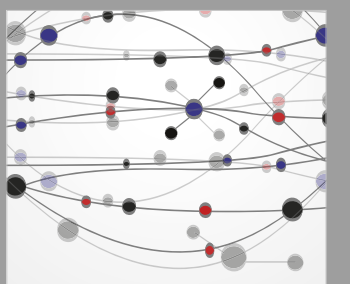

The Scientific World Journal
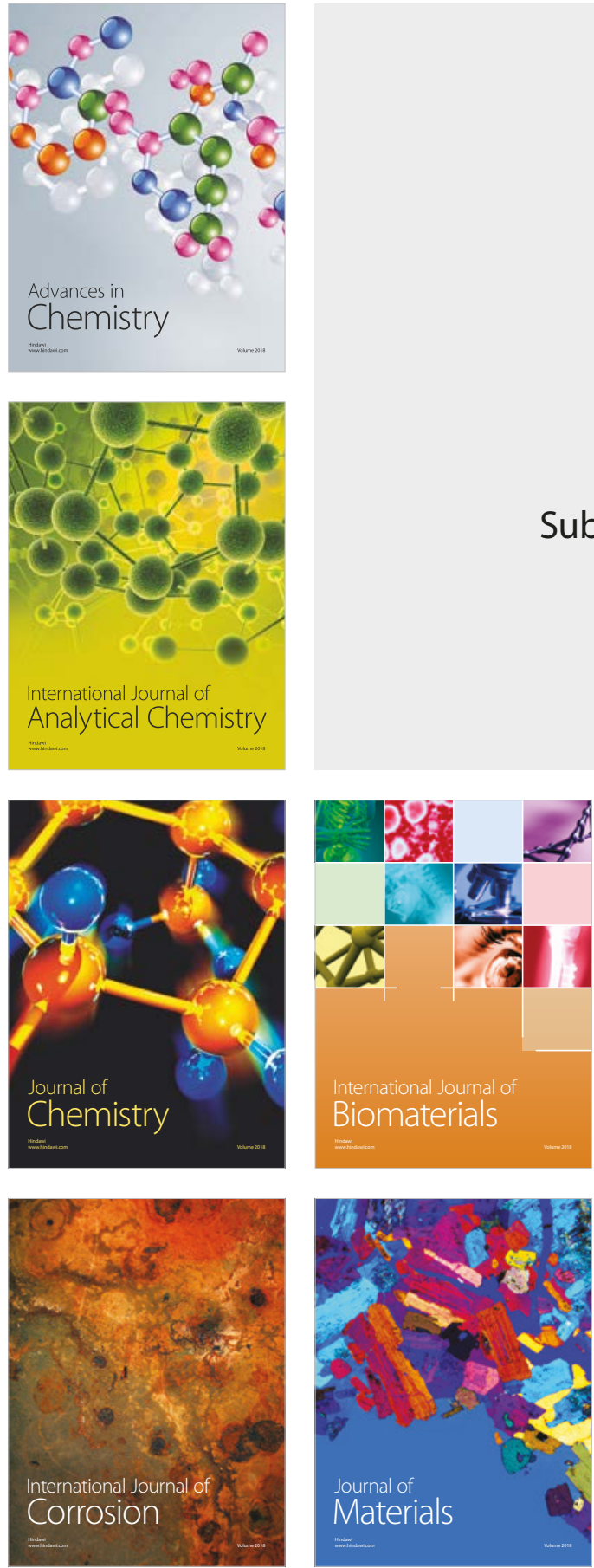

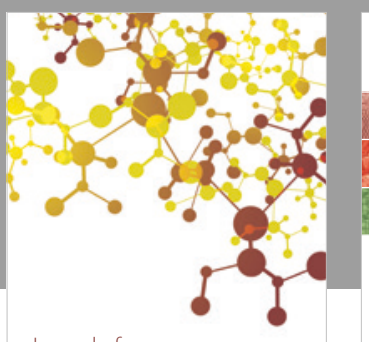

Journal of

Applied Chemistry
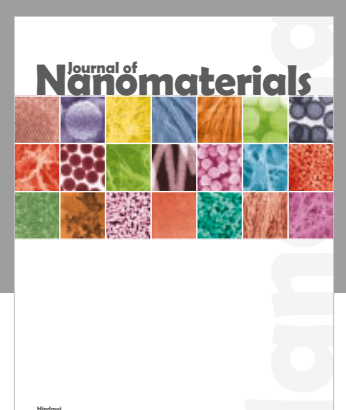

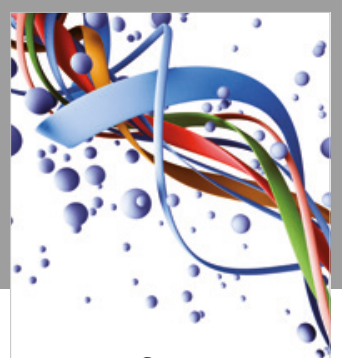

Scientifica

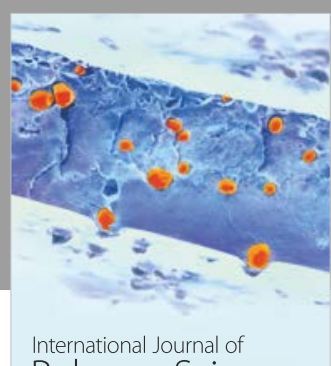

Polymer Science

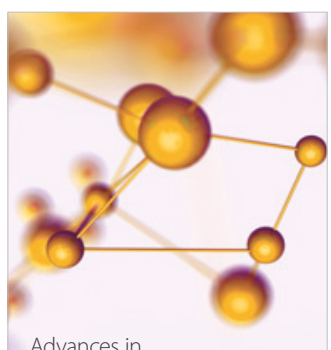

Physical Chemistry
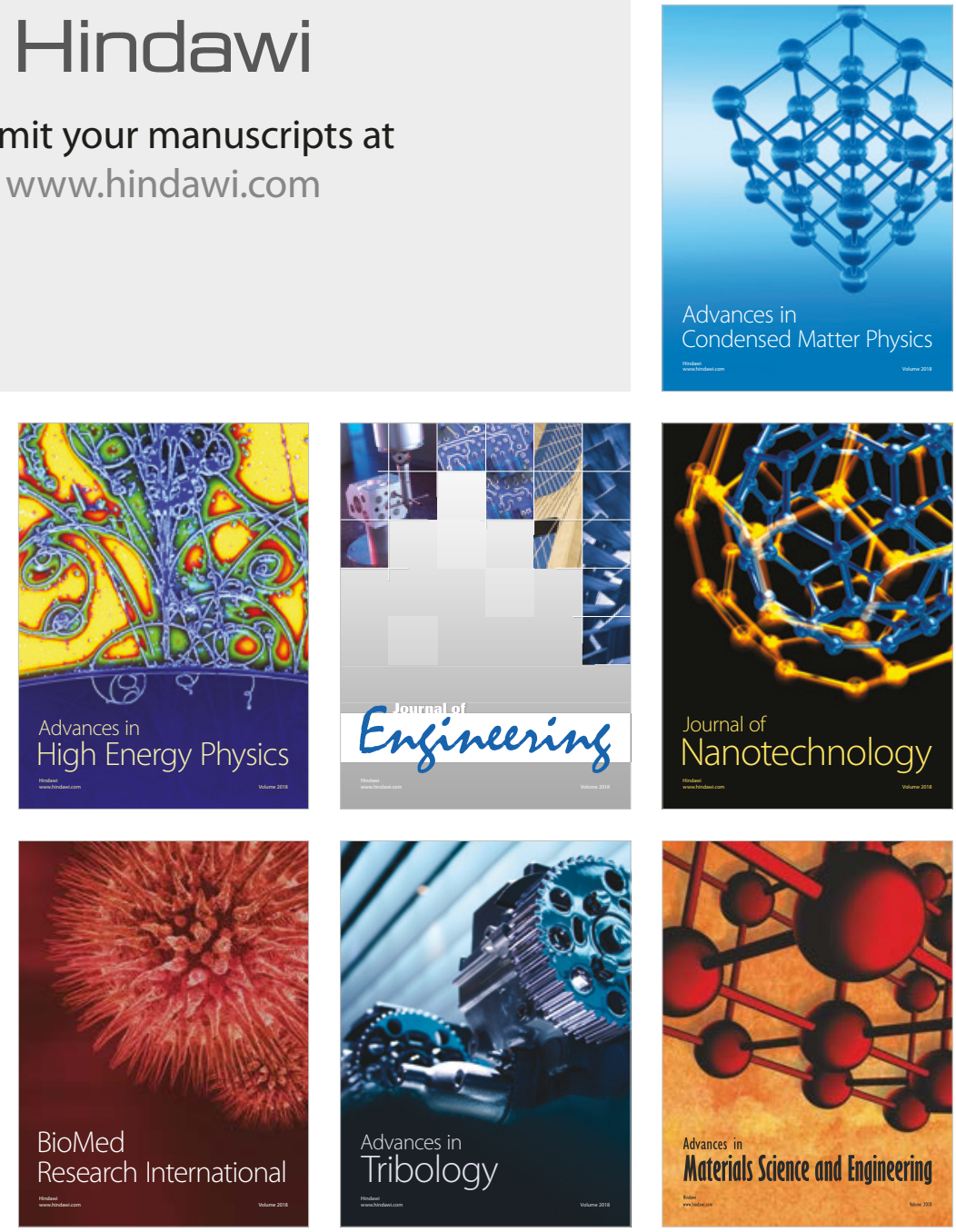